\title{
Foreword to special issue for ISAIM 2018
}

\section{Dimitrios I. Diochnos ${ }^{1}$ (1) . Jürgen Dix ${ }^{2}$ Guillermo R. Simari ${ }^{3}$}

Published online: 12 May 2020

(C) Springer Nature Switzerland AG 2020

During the first few days of every even year since 1990, the International Symposium on Artificial Intelligence and Mathematics (ISAIM) has taken place in Fort Lauderdale, Florida. ISAIM is a three-day event, where people who work in the broader field of Artificial Intelligence (AI) get together, present and discuss new directions in their research work, and it is often-times the case that new collaborations are born. ISAIM focuses on the foundations and mathematical methods of AI. Apart from the main track where papers from all angles of AI can be found, smaller sessions are also organized and in there one can find papers that belong to the specific topic of each such session. In particular, ISAIM 2018 had the following special-sessions beyond the main track: (i) Flow Optimization in Traffic Networks, (ii) Boolean and pseudo-Boolean Functions, (iii) Formalising Robot Ethics, (iv) Theory of Machine Learning, and (v) Applications of SAT. This special issue of the journal of Annals of Mathematics and Artificial Intelligence is devoted to ISAIM 2018 and selected papers that appeared either in the main track, or in one of the specialized tracks of the symposium. The selected papers presented here are full length versions which have undergone a standard refereeing process appropriate for journal papers. The papers accepted fall broadly under the areas of game theory or learning theory, and one of the papers is at the intersection of these two fields where online learning and regret analysis are being used. Below, we provide some brief summaries of the papers that we had the pleasure to host in this special issue.

Savaux, Vion, Piechowiak, Mandiau, Matsui, Hirayama, Yokoo, Elmane, and Silaghi, using game theory, explore how agents can compromise solution quality in a distributed

Dimitrios I. Diochnos

diochnos@ou.edu

Jürgen Dix

dix@tu-clausthal.de

Guillermo R. Simari

grs@cs.uns.edu.ar

1 School of Computer Science, University of Oklahoma, Norman, OK, USA

2 Department of Informatics, TU Clausthal, Clausthal-Zellerfeld, Germany

3 Department of Computer Science and Engineering (DCIC), Universidad Nacional del Sur, Bahía Blanca, Argentina 
constrained reasoning setting in favor of preserving privacy. This protection is obtained by adopting a utilitarian definition of privacy in the context of distributed constrained reasoning. They discuss implications, present a model and solvers, and ultimately show how privacy requirements in such a setting can be modeled as a planning problem, and more specifically as a stochastic game. Eventually, experiments are performed using different metrics in order to validate the approach that was adopted.

Feldbacher-Escamilla and Schurz explore probability aggregation using the lens of online learning with expert advice. In particular, various characterization results are known for probability aggregation rules which, however, have several parameters free and uninterpreted. They suggest interpreting these parameters for linear aggregation using success-based scores which are a generalization of Brier scoring. Working along this line, they are able to ultimately transfer optimality results that hold in the framework of online learning with expert advice, to the framework of probability aggregation.

Clémençon, Bertail, and Ciołek explore generalization results within learning theory when there are dependencies among the training data. In particular, they explore learnability when the training data are determined by a (Harris positive) Markov chain. In this direction, new concentration results are derived using the regenerative method, and these results depend both on the complexity of the class that is being learned as well as on the ergodicity rate of the chain. These results are then applied to the Minimum Volume sets problem, and eventually, with the aid of experiments, a better understanding of the theoretical results is obtained.

Mahloujifar, Diochnos, and Mahmoody explore poisoning attacks within probably approximately correct (PAC) learning, where the adversary has to respect the label of the ground truth for the adversarial instances. Initially, they explore the case where the adversary substitutes each training example with some fixed probability $p$, and there they show that PAC learning is still possible whenever it is possible in the realizable case in the absence of the adversary. Then they explore attacks where the adversary has the power to choose which $p$-fraction of the training examples to substitute, and in this setting, they show that PAC learning is no longer possible. As a remark, since one of the editors is also an author in this paper, we note that in order for all the authors of this paper to receive fair and anonymous reviews, the paper was handled by Jürgen Dix using a separate pool of papers where he was also serving as an editor at the time.

Giuliodori, Bistarelli, and Mugnai explore issues that arise in energy allocation and payment. In particular, they take a mechanism design approach for energy allocation among customers, so that by taking consumers' consumption profiles into account, no blackouts occur. Then, they design a payment mechanism using the concepts of Shapley values and explore its efficiency as an economic equilibrium among sellers and buyers, as well as its fairness as an economic measure of energy good-behaving.

Lange, Nguyen, and Rothe explore fair division of indivisible goods and consider settings where agents can have nonzero utility for the empty bundle. They first show that by allowing nonnormalized utility functions, computing an allocation that maximizes social welfare is computationally hard. Such a result contrasts known results for normalized utility functions where, under certain restrictions, efficient algorithms are available. Also, they show that maximizing elitist social welfare with $k$-additive utility functions is an NP-complete problem for every $k \geq 2$.

In closing, we would like to thank the authors of this special issue for their contributions, as well as the other participants in ISAIM 2018, the members of the program committee, and the invited plenary speakers, Cynthia Rudin and Craig Boutilier. Special thanks go to 
Martin Golumbic, Editor-in-Chief of the Annals of Mathematics and Artificial Intelligence Journal, and General Chair of ISAIM for his always available guidance. We would also like to thank Frederick Hoffman, who has helped yet again with the organization of a smooth and most enjoyable conference for all the participants in Fort Lauderdale, Florida.

Publisher's note Springer Nature remains neutral with regard to jurisdictional claims in published maps and institutional affiliations. 